\title{
SARS-CoV (COVID-19) Pandemic - Detailed Insights into Diagnosis, Management, and Role of Indian Herbal Drugs
}

\author{
Rahat Kumar ${ }^{1}$, Parminder Singh ${ }^{2}$, Richa G Thaman ${ }^{3}$, Rajiv Choudhari ${ }^{4}$, Simranjeet Kaur ${ }^{5}$
}

\begin{abstract}
Coronavirus-2019 (COVID-19) (SARS-CoV-2), a pandemic by World Health Organization on March 11, 2020, is caused by corona viruses which is highly contagious with enveloped positive-sense RNA particles with a size of 60-140 nm in diameter. The COVID-19 infection may be an asymptomatic, mild, moderate or severe illness with severe potentially lethal complications, such as severe pneumonia, respiratory failure, acute respiratory distress syndrome, sepsis, and septic shock due to induction of cytological storm. Diagnostic confirmation is by reverse transcription polymerase chain reaction. The treatment is mainly symptomatic with management of complications. Antiviral drugs like lopinavir administration have not been proven very useful and other drugs, such as Arbidol and favipiravir have some hope. Remdesivir, a drug approved by USFDA, has shown benefits in critical patients with COVID-19 infection. The mode of action of chloroquine and hydroxychloroquine is via immunomodulatory effects. Ministry of AYUSH has recommended intake of ayurvedic herbal drugs like curcumin, tulsi, cinnamon, black pepper, ashwagandha, shunthi (dry ginger), and regular yoga. Curcumin and garlic are important anticytokine agents and decrease various substances like interleukin (IL)-1 $\beta$, IL-10, and IL-12 by macrophages with additional fibrinolytic and antiplatelet aggregatory actions. Amla is an immunity booster. Tulsi due to phytocompounds and apigenin might be useful in COVID-19 infection. Cinnamomum has eugenol with antiviral activity. Black pepper suppresses the nuclear factor kappa-B signaling pathway. Ginger (Zingiber officinale) decreases various proinflammatory chemokines IL-8 and regulated upon activation, normal T-cell expressed and secreted. Mulethi has saponins which inhibit in vitro several DNA- and RNA-viruses. Thus, Indian herbal system of Ayurveda is very useful in COVID-19 infection.
\end{abstract}

Keywords: Ayurvedic drugs, COVID-19, Diagnosis, Herbal drugs.

AMEl's Current Trends in Diagnosis \& Treatment (2020): 10.5005/jp-journals-10055-0107

\section{INTRODUCTION}

Severe acute respiratory syndrome (SARS) due to coronavirus-2019 (COVID-19) was declared a pandemic disease by the World Health Organization on March 11, 2020. Coronaviruses (CoVs) are a group of viruses belonging to the Nidovirales class which are enveloped, nonsegmented, and positive-sense RNA viruses. ${ }^{1}$ CoVs has a size ranging from 60 to $140 \mathrm{~nm}$ in diameter with projections on the surface giving it a crown-like appearance called coronavirus. ${ }^{2} \mathrm{CoV}$ s are enveloped containing a nonsegmented, positive-sense RNA genome of $\sim 30 \mathrm{~kb}$ (size 26.2-31.7 kb). ${ }^{3}$ Four CoVs namely HKU1, NL63, 229E, and OC43 are known to infect humans. The shape of the coronavirus is either pleomorphic or spherical with clubshaped spiky projections of glycoproteins on its surface (diameter 80-120 nm). The coronavirus particles are made up of four structural proteins and these are spike $(S)$, membrane $(M)$, envelope $(E)$, and nucleocapsid $(\mathrm{N})$ proteins. All of these proteins are encoded within the $3^{\prime}$ end of the viral genome and $5^{\prime}$ end of the coronavirus genome has a leader sequence with multiple stem-loop structures required for RNA replication and transcription.

\section{Epidemiology and Pathogenesis}

Coronavirus after the infection is colonized in the nasopharynx causing fatal pneumonia was first reported in people of Wuhan, China on December 29, 2019. ${ }^{4}$ The basic reproduction number $\left(R_{0}\right)$ defined as "the expected number of cases directly generated by one case in a population where all individuals are susceptible to infection" is an important tool to estimate the spread of the coronavirus and is 2 to $6.47,{ }^{5}$ signifying the high contagiousness of COVID-19 infection.

\footnotetext{
1'Department of Pharmacology, Shri Guru Ram Das Institute of Medical Sciences and Research, Amritsar, Punjab, India

${ }^{2}$ Department of Medicine, Shri Guru Ram Das Institute of Medical Sciences and Research, Amritsar, Punjab, India

${ }^{3}$ Department of Physiology, Shri Guru Ram Das Institute of Medical Sciences and Research, Amritsar, Punjab, India

${ }^{4}$ Department of Forensic Medicine, Shri Guru Ram Das Institute of Medical Sciences and Research, Amritsar, Punjab, India

${ }^{5}$ Department of Microbiology, Government Medical College, Amritsar, Punjab, India
}

Corresponding Author: Richa G. Thaman, Department of Physiology, Shri Guru Ram Das Institute of Medical Sciences and Research, Amritsar, Punjab, India, Phone: +91 9815963778, e-mail: richaghaythaman@ yahoo.co.in

How to cite this article: Kumar R, Singh P, Thaman RG, et al. SARSCoV (COVID-19) Pandemic-Detailed Insights into Diagnosis, Management, and Role of Indian Herbal Drugs. AMEl's Curr Trends Diagn Treat 2020;4(2):95-102.

Source of support: Nil

Conflict of interest: None

\section{Risk of Transmission}

The source of the outbreak is not known but preliminary investigations show association of bats SARS-CoV-2 with Huanan Seafood Wholesale Market, Wuhan City of China. COVID-19 virus is primarily transmitted between people through respiratory droplets and through contact routes. The risk is directly related to type, duration of exposure, use of preventive measures, and 
individual immunological factors (e.g., innate immunity, disease states, etc.).

\section{IMMUNOPATHOGENESIS OF COVID-19 INFECTION}

SARS-CoV-2 infects the epithelial cells of the lungs after endocytosis and binding to the transmembrane angiotensin-converting enzyme-2 protein on cells in lungs, heart, blood vessels, kidney, and gastrointestinal tract. The virus enters the macrophages and dendritic cells and the infection may progress to induce proinflammatory cytokines. ${ }^{6}$ The scheme of pathogenesis is as below:

- Antigen presentation in coronavirus infection: After COVID19 infection, coronavirus antigen is presented to the antigen presentation cells (APCs) generating antigenic peptides presented to the major histocompatibility complex (MHC)-I. ${ }^{7}$

- Humoral and cellular immunity: APC stimulates the body's humoral and cellular immunity by viral-specific B- and T-cells. The antibody formation against SARS-CoV consists of immunoglobulin (Ig)M and IgG. The SARS-specific IgM antibodies present in body up to 12 weeks while protective IgG antibodies last for a much longer time with the duration not known. ${ }^{8}$

- Acute phase response in patients with SARS-CoV: SARS-CoV infection is associated with a severe decrease of CD4p-Tand CD8p-T cells and it has been seen that CD4p and CD8p memory T-cells can persist for four years in recovered patients and can perform T-cell proliferation, delayed-type hypersensitivity response, and production of interferon gamma (IFN-g). ${ }^{9}$

- Cytokine storm in COVID-19: Cytokine storm is a potentially lethal complication leading to complications, such as acute kidney injury (AKI), acute lung injury (ALI)/fibrosis, pancreatitis, and cardiac injury. Cytokine storms are manifested by high plasma levels of various proinflammatory cytokines like interleukin (IL)-2, IL-7, IP10, monocyte chemoattractant protein-1 (MCP-1), macrophage inflammatory protein 1- $\alpha$ (MIP1A), granulocyte colony-stimulating factor, and tumor necrosis factor- $\alpha$ (TNF- $\alpha) .{ }^{10}$ In addition, a series of inflammatory chemokines (CC), such as CCL2, CCL3, CCL5, and CCL11 and IFN- $\gamma$-responsive CC like C-X-C motif ligand (CXCL)1, CXCL8, CXCL9, and CXCL10 are generated. ${ }^{11}$ It has been seen that the CCL3, CCL5, and CXCL10 CC contribute to a lung pathology via triggering the coagulation system due to activation and migration of the cytotoxic T-lymphocytes leading to multiorgan damage. ${ }^{12}$

- Pulmonary fibrosis (PF) in COVID-19 infection: Adipocytemyofibroblast transition and pulmonary lipofibroblasts present in the alveolar interstitium transdifferentiate into myofibroblasts contributing to PF and diffuse alveolar damage. ${ }^{13}$ Research also indicates that respiratory failure in COVID-19 is due to microvascular thrombosis in pulmonary blood vessels and thus both lung injury and thrombosis might play a role in the pathogenesis of viral infections. ${ }^{14}$

- SARS and multiorgan failure (MOF): MOF is due to stimulation of secretion of a large number of inflammatory particles from arachidonic acid pathway, such as IL, IFN, CXCL chemokine producing cytokine cascade reaction. ${ }^{14}$ Therefore, the inhibition of amino acid metabolism pathway is important for inhibiting "cytokine storms."

\section{Age and Transmission}

Globally, >5 million confirmed cases of COVID-19 have been reported. The median time from the onset of symptoms to dyspnea is 5 days, to hospitalization is 7 days, and to acute respiratory distress syndrome (ARDS) is 8 days. The mode of spread is via respiratory droplets and the droplets can travel up to $6 \mathrm{ft}$ and can remain viable up to 7 days but are destroyed in a minute by disinfectants like sodium hypochlorite, hydrogen peroxide, and $60-70 \%$ alcohol. $^{15}$ The transplacental transmission from pregnant women to their fetus has not been established.

\section{Clinical Features}

The COVID-19 infection may present with a mild, moderate or severe illness or may progress to pneumonia, respiratory failure, and death. The presentation of patients is as follows:

- Uncomplicated (mild) Illness: Upper respiratory infection like mild fever, persistent dry cough, severe pain in throat, nasal/eye congestion, malaise, headache, and muscle pain.

- Moderate Illness: In addition to the above, have respiratory problems, such as dyspnea (or tachypnea) but without signs of severe pneumonia.

- Severe Illness:Unexplained dyspnea, respiratory distress, tachypnea (respiratory rate $>30$ breaths/minute), and mild-to-moderate hypoxia (hemoglobin saturation $\left(\mathrm{SpO}_{2}\right)<90 \%$ on room air).

- Acute respiratory distress syndrome (ARDS): The diagnosis is a clinical degree of hypoxia based on partial pressure of oxygen in the arterial blood $\left(\mathrm{PaO}_{2}\right) /$ the fraction of inspired oxygen $\left(\mathrm{FiO}_{2}\right)$ ratio: ${ }^{16}$

o Mild ARDS: $\mathrm{PaO}_{2} / \mathrm{FiO}_{2}$ is $200-\leq 300 \mathrm{~mm} \mathrm{Hg}$. In patients on oxygen mask or with noninvasive ventilation by using positive end-expiratory pressure or a continuous positive airway pressure: (CPAP) $\geq 5 \mathrm{~cm} \mathrm{H}_{2} \mathrm{O}$.

o Moderate ARDS: $\mathrm{PaO}_{2} / \mathrm{FiO}_{2}$ is $100 \mathrm{~mm} \mathrm{Hg}$ to $\leq 200 \mathrm{~mm} \mathrm{Hg}$.

o Severe ARDS: $\mathrm{PaO}_{2} / \mathrm{FiO}_{2}$ is $\leq 100 \mathrm{~mm} \mathrm{Hg}$.

However, when $\mathrm{PaO}_{2}$ is not available, a ratio of $\mathrm{SpO}_{2} / \mathrm{FiO}_{2} \leq 315$ is suggestive of ARDS.

\section{Complications and Clinical Outcomes}

Recovery after infection is $2 \mathrm{nd}$ or 3 rd week with median duration of hospital stay is 10 days. The overall case fatality rate ranges between $2 \%$ and $3 \%$ and is seen in patients with serious complications, such as duration of exposure to viral antigen, ARDS, arrhythmia, shock, $\mathrm{AKI}$, acute cardiac injury, liver dysfunction, and secondary infection.

\section{Diagnostic Criteria}

World Health Organization (WHO) recommends collection of specimens from both upper and lower respiratory tracts, such as expectorated sputum, endotracheal aspirate or bronchoalveolar lavage. After collection, amplification of the genetic material is done through a reverse transcription polymerase chain reaction (RT-PCR) ${ }^{17}$ and the sensitivity of this test is $50 \%$ to $80 \%$. Other nonspecific laboratory investigations are white cell count (normal or low), lymphopenia (count $<1000 \mathrm{cu} / \mathrm{mL}$ ), normal or mildly low platelet count, high C-reactive protein (CRP), and erythrocyte sedimentation rate and procalcitonin levels elevated in bacterial sepsis or septic shock. High alanine transaminase /aspartate aminotransferase, prothrombin time, creatinine, D-dimer, creatine phosphokinase, and lactate dehydrogenase directly relate with the level of injury in the 
patient and is associated with poor prognosis. ${ }^{18}$ High levels of IL-6 is predictive of a cytological storm. IL- 6 can bind to transmembrane IL-6 receptors (mIL6R) and soluble IL-6 receptors (sIL-6R) resulting in formation of a complex can combine with a signal-transducing component gp130 of coronavirus producing further activation of inflammatory trigger. ${ }^{19} \mathrm{X}$-ray chest at this early stage may show multiple small patchy shadows with interstitial changes showing bilateral multiple ground-glass opacities and infiltrations with consolidation at peripheral and basal areas of the lungs as shown by CT scan at this stage. That is why abnormal CT scans have been used to diagnose COVID-19 in suspected cases with negative molecular diagnosis. For patients with acute fever $\left(>37.5^{\circ} \mathrm{C}\right)$, an absolute count of peripheral lymphocytes is less than $0.8 \times 109 / \mathrm{L}$ or a falling count of CD4+ and CD8+ T-cells needs isolation and close observation. In these cases, a repeat of real-time reverse-transcription polymerase chain reaction (rRT-PCR) or chest CT scan should be done.

\section{Investigation of the Patient with SARS- COV-2 InfECtION}

The following schemes are used for patients having infection: ${ }^{20}$

- Screening of patient on the day of presentation: After complete blood count (CBC), urine analysis, liver/kidney function (LFT/ KFT), CRP, nucleic acid examination (RT-PCR) of sputum or naso/ oropharyngeal secretions should be done to confirm the case and in case of nonavailability of RT-PCR, then patient should be labeled as suspected case. Other tests can be done such as arterial blood gas analysis (ABG), serum lactate, procalcitonin (PCT), creatine kinase plus myoglobin, coagulation profile, such as BT/CT, X-ray chest, and chest CT. Additionally, inflammatory cytokines [such as IL-6, IL-10, and tumor necrosis factor (TNF)- $\alpha$ ], tuberculosis (TB) lymphocyte subsets, and complement can be tested for severity estimation. The laboratory data showed that the critical intensive care unit (ICU) patients had higher plasma levels of IL-2, IL-7, IL-10, granulocyte colony-stimulating factor (GCSF), $10 \mathrm{kD}$ interferon gamma-induced protein (IP-10), monocyte chemoattractant protein-1 (MCP-1), macrophage inflammatory protein $1-\alpha$ (MIP-1 $\alpha$ ), and TNF- $\alpha{ }^{21}$

- Sequential examination of confirmed patients: The sequential examination of the patient is as follows: $C B C$, coagulation function$\mathrm{BT} / \mathrm{CT}$ and CRP, LFT/KFT, creatine kinase and myoglobin checked on the 3rd, 5th, and 7th days, and PCT and neutrophil-lymphocyte count ratio (NLCR) can be repeated on days 5-7 if required.

\section{Management of Patients with COVID-19 INFECTIONS}

The management of patients is as follows:

\section{Prevention}

WHO has issued various recommendations as below:

- Avoid close contact with patients having any acute respiratory infections.

- Wash hands, nails, and interdigital areas frequently with soap and water for 20 seconds.

- Avoid frequent touching of face, nose, and mouth.

- Avoid unprotected contact with pets or farm or wild animals.

- Coughs or sneezes with elbow, disposable tissues or clothes.

- Immunocompromised subjects like elderly aged $>65$, children less than 10 years, and pregnant patients should avoid going to public gatherings.
- Healthcare workers must wear PPE kit, N95 or FFP3 masks, eye protection/shield, gowns, and gloves.

\section{Treatment of Infected Patients}

The treatment is mainly symptomatic and oxygen therapy is most important for patients with severe infection. Mechanical ventilation like CPAP, intermittent positive-pressure ventilation (IPPV), and invasive ventilator may be required in cases of respiratory failure.

\section{Oxygen Therapy}

Hypoxic patients should be given oxygen therapy immediately on arrival at hospital. The blood oxygen saturation level should be kept $>90 \%$ in men and nonpregnant women and between $92 \%$ and $95 \%$ in pregnant women with oxygen $5 \mathrm{~L} /$ minutes by nasal cannula. If the patient is still dyspneic or is getting deteriorated, then a highflow nasal cannula should be used with oxygen supplementation at the rate of $20 \mathrm{~L} /$ minutes, increasing gradually to 50 to $60 \mathrm{~L} /$ minutes based on oxygen saturation, i.e., by ABG.

\section{Intubation and Protective Mechanical Ventilation}

Noninvasive ventilation should be supplemented by $100 \%$ oxygen via CPAP. Patients requiring invasive ventilation and endotracheal intubation should be performed by an intensivist. Mechanical ventilation may be used in patients with lower tidal volumes (4-6 $\mathrm{mL} / \mathrm{kg}$ predicted body weight) and patients with lower inspiratory pressures reaching a plateau pressure (Pplat) $<28-30 \mathrm{~cm} \mathrm{H} \mathrm{H}_{2} \mathrm{O}$. The prone ventilation for $>12$ hours/day and conservative fluid management for ARDS patients without tissue hypoperfusion are recommended.

\section{Chloroquine and Hydroxychloroquine}

These agents are also called as disease-modifying antirheumatic drugs (DMARDs). The exact mode of action of DMARDS in COVID19 infection is not clear but it has been postulated that both the agents being weak bases accumulate in acidic organelles of human cells like lysosomes thereby increasing the endosomal/ lysosomal $\mathrm{pH}$ that inhibits viral replication. ${ }^{22}$ Both the agents have been shown to inhibit major expression of MHC-II, antigen presentation, and immune activation by reducing CD154 expression by T-cells via Toll-like receptor signaling and cGAS (cyclic GMP-AMP synthase) stimulation and thus reduce the production of cytokines responsible for generation of cytological storm, such as IL-1, IL-6, IFN- $\alpha$, and TNF- $\alpha .{ }^{23}$ Dosage: hydroxychloroquine is (200 mg every 12 hours). The prophylactic dose of hydroxychloroquine is $400 \mathrm{mg}$ bd on day 1 and $400 \mathrm{mg}$ once a week for the next 3 weeks to be taken after meals.

\section{Antiviral Agents in COVID-19 Infections}

No specific antiviral treatment is approved. The various proposed antiviral agents are as follows:

- Lopinavir (400 mg)/ritonavir (100 mg) orally every 12 hours for 5-21 days until patient's coronavirus is detected negative by RT-PCR for consecutive three times. However, Jean et al., ${ }^{24}$ concluded in their study that the protease inhibitor lopinavir/ ritonavir (LPV/RTV) alone does not provide better antiviral efficacy than standard care. In a randomized clinical trial ${ }^{25}$ on 99 patients with severe COVID-19 illness, 50 patients were given LPV/RTV (400/100 mg twice a day) and the rest were given only standard of care for 14 days. The results of the study indicate that LPV/RTV was not associated with a statistically significant difference in time to clinical improvement. 
- Ribavirin is administered via intravenous infusion at a dose of $500 \mathrm{mg}$ for adults, two to three times/day in combination with IFN- $\alpha$ or LPV/RTV.

- Arbidol (ARB) - a Russian-made potent broad-spectrum has mode of action by inhibition of virus-mediated fusion with host target membrane and hence resulting in blockage of virus entry into target cells. Zhu et al., ${ }^{26}$ in 50 patients with COVID-19 infection had shown that patients given $200 \mathrm{mg}$ Arbidol, three times a day had a lower viral load than LPV/RTV. This concluded that ARB monotherapy was superior to LPV/RTV therapy in treating COVID-19. ARB is orally administered at a dose of $200 \mathrm{mg}$ for adults, 3 times/day for no more than 10 days.

- Favipiravir is approved for treatment of novel influenza and has a mode of action of inhibition of viral RNA-dependent RNA polymerase (RdRp) due to being converted into an active phosphoribosylated form (favipiravir-RTP) in viral cells. ${ }^{27}$ Although not much clinical data are available at present about the safety and efficacy of favipiravir, the results of ongoing future trials might show the efficacy in patients.

- Remdesivir (RDV): Remdesivir is the drug that was initially designed for the treatment of Ebola hemorrhagic virus (EBV) and Marburg virus infections and is being approved by USFDA for its effectiveness against 2019-nCoV infection. RDV is a nucleotide analog inhibitor of RNA-dependent RNA polymerases (RdRps) and can inhibit replication of multiple coronaviruses in nasal and respiratory epithelial cells. RDV is a prodrug of nucleoside analog with broad-spectrum antiviral activities against RNA viruses, including SARS-CoV and Middle East respiratory syndrome (MERS-CoV). RDV is converted to active adenosine nucleotide triphosphate analog which interferes with the action of viral RdRp and thus evades proofreading by viral exoribonuclease (ExoN) causing a decrease in viral RNA replication. In an experimental study, ${ }^{28}$ RDV given 1 day after the disease decreased lung virus titers with improvements in pulmonary function. After 2 days of infection, the pulmonary virus titer was reduced significantly, but the survival rate of mice was still low. This shows that RDV if given in early stages can prevent lung injury induced by viral replication. The exact dose of RDV is not available but is given as an initial dose of $200 \mathrm{mg}$ stat on the first day followed by a maintenance dose of $100 \mathrm{mg} /$ day up to a maximum of 10 total days of treatment.

- Other antiviral agents: A joint research team at Shanghai Institute of Materia Medica and Shanghai Tech University in China performed drug screening and the team concluded that $>30$ antiviral drugs are available with potential antiviral activity against SARS-CoV-2. ${ }^{29}$ These agents are as indinavir, saquinavir, fosamprenavir, enzaplatovir, carfilzomib, ritonavir, lopinavir, remdesivir, raltegravir, montelukast, deoxyrhapontin, polydatin, carmofur, shikonin, ebselen, atazanavir, darunavir, tipranavir, presatovir, abacavir, bortezomib, elvitegravir, maribavir, chalcone, disulfiram, tideglusib, PX-12, TDZD-8, cyclosporin A, and cinanserin.

\section{Tocilizumab}

It is a monoclonal antibody that blocks IL-6 and is recommended for severe or critically ill patients with extensive bilateral lung lesions. Its absolute recommendation is COVID-19 infection with massive pulmonary infiltrative lesions causing severe lung injury (ALI) and ARDS. ${ }^{29}$ The exact dose is not defined but tocilizumab may be given at a dose of $8 \mathrm{mg} / \mathrm{kg}$ intravenously (IV) once a day. If no clinical improvement occurs, an additional dose up to a maximum of three doses with an 8-hour gap is given in consecutive doses.

\section{Ulinastatin}

It is a serine protease inhibitor with anti-inflammatory and antiIL-6 properties. ${ }^{30}$ It is approved by Drug Controller of India (DCGI) for the treatment of acute pancreatitis and sepsis. Ulinastatin is recommended in COVID-19 infection patients with exacerbated lung lesions. ${ }^{31}$ The benefits of ulinastatin is mainly due to inhibition of inflammatory cascade by reducing the elevated TNF- $\alpha, I L-6$, and IL-8, the activation of neutrophils, blocking nuclear factor-KB pathway which are mediators of proinflammatory process, downregulating chemokines (e.g., IL-8, macrophage inflammatory protein-2), and increasing neutrophil apoptosis. ${ }^{32}$ Dosage: The exact dose is not available but ulinastatin is given in a dose of 200,000 units twice daily IV for 5 days.

\section{IFN- $\alpha$}

It is a broad-spectrum endogenously produced antiviral agent used to treat hepatitis and additionally is reported to inhibit SARSCoV reproduction in vitro. ${ }^{33}$ The dosage is via vapor inhalation of 5 million $\mathrm{U}$ (and $2 \mathrm{~mL}$ of sterile water for injection) for adults, 2 times/day.

\section{Intravenous Immunoglobulin}

Early IV infusion of human-pooled immunoglobulin is recommended for critically ill patients at a dose of $0.25-0.5 \mathrm{~g} /(\mathrm{kg} / \mathrm{d})$, for 3-5 days, ${ }^{34}$ but the efficacy is not known till date.

\section{Convalescent Plasma}

In India and the United States (Food and Drug Administration), USFDA has accepted it as an investigational new treatment therapy for severe or life-threatening COVID-19 patients with the benefit being described in case series. In one case series, ${ }^{35}$ the benefit of administration of donor plasma of patients recovered from COVID-19 infection in patients with severe COVID-19 infection on mechanical ventilation was seen. Patients reported a decreased nasopharyngeal viral load, disease severity score, and improved oxygenation by 12 days after transfusion.

\section{Glucocorticoid Therapy}

Glucocorticoids like prednisolone if given early in severe illness have been shown to provide benefit. IV methylprednisolone $40-80 \mathrm{mg}$ od for 5 days or more is recommended.

\section{Empirical Antimicrobial Therapy}

If a bacterial infection is suspected, antibiotics can be given depending on the patient's clinical, bacteriological, and imaging of chest findings. Patients with mild infection can take oral antibiotics. Patients with community-acquired pneumonia can be given cephalosporin or fluoroquinolones but in case of severe/critical infection, broad-spectrum antibiotics should be given.

\section{Role of Ivermectin in COVID-19 Infection}

Ivermectin antiparasitic drug has a prophylactic role in COVID-19 infection at a dose of $0.15 \mathrm{mg} / \mathrm{kg}-0.2 \mathrm{mg} / \mathrm{kg}$ body weight. The antiviral properties of ivermectin are well proven in RNA and DNA as well in BK polyomavirus and Equine herpesvirus infection. ${ }^{36}$ The probable mode of action is by inhibition of viral IMP $\alpha / \beta 1$-mediated nuclear import, which reduces the replication of virus and viral load due to creation of an acidic environment in virus. ${ }^{37}$ In a study by Wagstaff et al., ${ }^{38}$ ivermectin was demonstrated to have a potent in vitro inhibitor activity against severe acute respiratory syndrome coronavirus 2 (SARS-CoV-2) showing a 99.8\% reduction in viral RNA 
load after 48 hours of infection. However, the clinical usefulness of ivermectin in SARS-CoV-2 infected patients is unpredictable at this point as well-designed, large scale randomized, controlled clinical trials are needed to establish the efficacy of ivermectin in SARS-CoV-2 infection.

\section{Criteria of Isolation Release and Discharge of Patients ${ }^{39}$}

Patients can be discharged if serologically negative and has the following findings, such as normalization of body temperature persistently for $>3$ days, improvement of breathlessness, resolving of the pulmonary lesions on X-ray chest/CT scan, and negative RT-PCR for two consecutive times at least 1 day apart.

\section{Plant-based Therapy in COVID-19 INFECTIONS}

Medicinal herbs are being used for a variety of viral illnesses like influenza, hepatitis, chicken pox, herpes, HIV, etc. The potential plant-based drugs in the treatment of COVID-19 infection are:

- Star anise (Illicium verum): It is an evergreen, medium-sized tree with star-shaped fruit, with wide distribution throughout the southwestern parts of the Asian continent with antiviral effects. It is also the source of the precursor molecule, shikimic acid, which is used in the manufacturing of oseltamivir (Tamiflu ${ }^{\circledR}$, an antiviral drug for influenza $A$ and influenza B. ${ }^{40}$

- Chinese herbal formulae: ${ }^{41}$ In China, 17 herbal formulae with a slight difference in the herbal compositions are used. Some of these are Shen Fu Tang with Su He Xiang pill or Angong Niuhuang pill. The herbal formula of Qingfei Paidu Tang (a combination of four different herbal formulae with 21 herbs).

- Chinese drugs preventing cytological storm: The traditional Chinese medicine database 2009 (TCMD 2009) has ranked Huoxiang, Sparstolonin B (SsnB), and Sparstolonin B as examples for "cytokine storm" inhibiting the expression of various inflammatory mediators, such as TNF- $\alpha$ ), interleukin (IL)-1 $\beta$, IL-6, and chemokine (C-C motif) ligand 2 (CCL-2). ${ }^{42}$

\section{Indian Guidelines against COVID-19 INFECTION}

Ministry of AYUSH has recommended the following guidelines:

\section{Recommended Measures in the Prevention/Treatment of COVID-19 Infection}

\section{General Measures}

These include drink frequently warm water throughout the day, daily practice of yogasana, especially Pranayama and meditation for at least 30 minutes, enhance the consumption of food supplement spices like dhaniya (coriander), haldi (turmeric), jeera, and lahsun (garlic) in cooking or as a pickle.

\section{Ayurvedic Immunity-promoting Herbs}

Chyawanprash $10 \mathrm{~g}$ (1 tsf) twice a day morning and evening. Diabetics can take sugar-free Chyawanprash. Drink herbal tea mixture/decoction (kadha) made up of various herbs, such as dalchini (cinnamon), tulsi (basil), kalimirch (black pepper), shunthi (dry ginger) and munakka (raisin) - once or twice a day, golden milk-take half teaspoon full of haldi (turmeric) powder in $150 \mathrm{~mL}$ hot milk_once or twice a day.

\section{Yoga-based Procedures}

These should be tried only with expert advice and these include nasal application of sesame oil/coconut oil or ghee in both nostrils (Pratimarsha nasya) morning and evening, oil-pulling therapyhere swishing of oil with 1 tablespoon of sesame or coconut oil in mouth is done to remove bacteria and promote oral hygiene.

\section{During the Episodes of Dry Cough/Sore Throat}

Steam inhalation with fresh pudina (mint) leaves or ajwain (caraway seeds) once a day, lavang (clove) powder in small amount (5-10 g) mixed with natural sugar/honey taken 2-3 time/day.

\section{Other Measures}

The holistic approach of AYUSH system of medicine given with lifestyle modification, dietary management, and prophylactic interventions promotes immunity.

\section{Medical Evidence of Indian Herbs in COVID-19 Infection}

Health experts have suggested that boosting the body's immune system with some of the herbs may minimize the adverse outcome and these are tulsi, cinnamon, black pepper, and shunthi (dry ginger). The Indian herbs with potential benefits are described below:

- Curcumin: The main component of Indian spice turmeric (Curcuma longa) has many useful properties like antiinflammatory, antiangiogenesis, and antimicrobial. In a study by Sornpet et al., ${ }^{43} \mathrm{C}$. longa was shown to have strong antiH5N1 activity in the Madin-Darby canine kidney cells showing potential therapeutic benefit for the treatment of H5N1 infection. Curcumin is a powerful anticytokine agent and the various immunomodulatory actions of curcumin ${ }^{44}$ are:

o Down-regulating the activity of cyclooxygenase-2 (COX-2), lipoxygenase, and inducible nitric oxide synthase (iNOS) enzymes.

o Inhibition of inflammatory cytokines, such as tumor necrosis factor-alpha (TNF- $\alpha$ ), interleukin (IL)-1, IL-2, IL-6, IL-8, and IL-12, and MCP.

o Down-regulating mitogen-activated and Janus kinases, COX-2, and iNOS inhibition via suppression of nuclear factor kappa B (NF-KB) activation.

o Suppression of NF-KB activation and proinflammatory gene expression by blocking phosphorylation of inhibitory factor I-kappa B-kinase (ІкB).

o Reduction of multiple factors of inflammation, such as cytokines, TNF- $\alpha$, IL- 6 , and C-reactive protein (CRP) levels in blood serum.

o Thus, it can be interpreted that curcumin administration as a prophylactic or even during disease process may halt or slow down the cytological storm in COVID-19 patients.

- Amla (Syn. Emblica officinalis Gaertn.) or Chyawanprash: Plant products of Phyllanthus emblica L. family are traditionally consumed in India and various parts of the world for their many therapeutically beneficial activities like immune building, antidiabetic, antioxidant, antimicrobial, antifungal, antiallergic, antiviral, and anticancer properties. A study by Liu et al., ${ }^{45}$ showed that the pentagalloylglucose (PGG) obtained from $P$. emblica had anti-influenza virus activity. The findings in the same study indicate that PGG treatment inhibited 
influenza-virus-induced hemagglutination of chicken red blood cells suggesting activity of PGG against IAV (influenzaassociated virus). Thus, regular use of amla may prevent attachment of the coronavirus protein to the host cells and thereby may be used as prophylaxis for viral infections.

- Tulsi (holy basil) or Ocimum sanctum: $O$. sanctum L. is a plant which is seen in different parts of the world and is known to have medicinal properties. Tulsi is known to possess various properties like neuroprotective, cardioprotective, antibacterial, antifungal, antianaphylactic, antihistaminic, mast cell stabilizing, radioprotective, wound healing, antidiabetic, antioxidant, immunologic, anticancer, contraceptive, antigenotoxic, etc. O. sanctum has a variety of phytocompounds like eugenol, carvacrol, urosolic acid, linalool, limatrol, methyl carvicol, caryophyllene, anthocyanins, sitosterol, and apigenin. Alhazmi, ${ }^{46}$ observed that molecular docking of apigenin (from O. sanctum) with H1N1 proteins had inhibitory effects as compared to oseltamivir and zanamivir concluding that O. sanctum may be useful in treating COVID-19 infections.

- Dalchini (cinnamon): Cinnamomum (family Lauraceae) is used as a cooking and flavoring agent in all parts of India and has a protective action against cough and cold. Cinnamomum contains eugenol which is shown to have multiple antiviral activities against viruses like herpes simplex virus type 1 and 2 , tomato yellow leaf curl virus, feline calicivirus, influenza $A$ virus, and four airborne viruses. ${ }^{47}$ In a study by Yeh et al., ${ }^{48}$ Cinnamomum cassia extract was shown to inhibit human respiratory syncytial virus (HRSV) induced plaque formation in both human upper (HEp-2) and lower (A549) respiratory tract cell lines. Cinnamomum also has the ability to inhibit the synthesis of viral fusion (F) protein. C. cassia has the potential to prevent the airway epithelia from HRSV infection by inhibiting viral attachment, internalization, and syncytium formation.

- Kalimirch (black pepper or Piper longum L.): It is a very useful herbal bioenhancer of different classes of drugs. Active compounds from Piper plant extracts have been shown to have many properties like antiproliferative, anti-inflammatory, and neuroimmunomodulating. There are also reports of antioxidant effects of piperine extracts against a series of reactive oxygen and nitrogen species, such as scavenging of superoxide anion, hydrogen peroxide, nitric oxide, $\alpha,-\alpha$-diphenyl- $\beta$-picrylhydrazyl (DPPH) free radical, ABTS ( 2,2 '-azino-bis-3-ethylbenzthiazoline6-sulphonic acid)) and reducing effect against ferric and molybdenum. Antimicrobial activity has been shown in a wide range of pathogens and the proposed mode of action is inhibition of bacterial swarming, biofilm formation, bacterial efflux pumps, and swimming motilities. Piperine has also been shown to reduce the expression of inducible nitric oxide synthase, cyclooxygenase-2, proinflammatory cytokines, such as tumor necrosis factor (TNF)- $\alpha$ and interleukin-6 (IL-6), and nuclear factor kappa B (NF-KB) inducing signaling pathway. ${ }^{49}$ Thus, it can be interpreted that piperine administration may have beneficial effects in patients having corona infection due to inhibition of the culprit IL-6 produced during cytological storm.

- Garlic (Allium sativum L.): It is a well-known pungent, aromatic herb plant consumed worldwide. Garlic is known in Indian literature for its antimicrobial and antiviral properties and is used in Indian Ayurveda for the relief of common cold. Garlic is rich in several sulfur-containing phytoconstituents, such as alliin, allicin, ajoene, vinyldithiins, and multiple flavonoids mainly quercetin. ${ }^{50}$ In the experimental studies in Swiss mice, garlic has been shown to inhibit various cytokines' production like IL-1 1 , IL-10, and IL-12 by macrophages. ${ }^{51,52}$ Infectious bronchitis virus (IBV), a type of coronavirus, was shown to be inhibited experimentally in embryonic specific-free antigen (SPF) eggs added with garlic in the chicken's embryo demonstrating the inhibitory effects on IBV. ${ }^{53}$ Antithrombotic activities of odorless garlic powder are well documented. The fibrinolytic and anticoagulation mode of action is accelerating the t-PA-mediated plasminogen activation and also directly suppressing the coagulation system by downregulating thrombin formation in a rat in situ loop model. Thus, garlic has the potential to prevent thrombus formation due to its antiplatelet aggregation, lower the plasma fibrinogen level, and increase the fibrinolytic activity which is an important cause of high mortality in COVID-19.

- Ginger: Ginger (Zingiber officinale R.) is a common and also a widely used medicinal plant in food supplementation. The therapeutic benefits of ginger are due to the presence of many ingredients like oleoresin, volatile oils, phenols, and alkaloid. In a study ${ }^{54}$ conducted on bronchial epithelial cells (BEAS-2B cells) of humans using the extract of $Z$. officinale, inflammation was induced by lipopolysaccharide (LPS) and the effect of zinger was seen on various proinflammatory chemokine interleukin- 8 (IL-8) and regulated upon activation, normal T-cell expressed and secreted. The oily extract of the ginger rhizome was shown to reduce the LPS-induced IL-8 secretion suggesting that the ginger extract has anti-inflammatory effects on bronchial epithelial cells. ${ }^{55}$ In another study on 36 newborn Wistar rats, ginger extract was shown to reduce the lung damage from severe damage due to hyperoxia and inflammation. ${ }^{56}$ In the same study, it was seen that tissue TNF- $\alpha$, IL- $1 \beta$, and IL- 6 concentrations were decreased in the animals treated with ginger in comparison to the placebo group. Ginger constituents like zingerone, a phenolic alkanone isolated, was shown to suppress LPS-induced kidney damage (AKI) like lowering of BUN, creatinine, and inflammatory cytokines TNF- $\alpha$, IL- 6 , and IL-1 $\beta$ levels in a dose-dependent manner in mice.

- Mulethi (licorice root): The name "licorice" is derived from the ancient Greek word Glycyrrhiza meaning "sweet root." Licorice consists of approximately 30 species. Glycyrrhizic acid (compound I, GA) is the main saponin of licorice root (Glycyrrhiza glabra L. and Glycyrrhiza uralensis F.). Licorice extract and its monoammonium salt glycyram were shown to inhibit in vitro several DNA- and RNA-viruses (herpes simplex, vaccinia, Newcastle, vesicular stomatitis, herpes $B$, and varicella zoster). The mechanism of glycyrrhizin's activity against SARS-CoV-2 is unclear; however, glycyrrhizin has been shown to decrease the cellular signaling pathways like protein kinase-C, casein kinase II, and various transcription factors, such as activator protein 1 and nuclear factor $\mathrm{KB} .{ }^{57}$ In a study by Kim et al., ${ }^{58} \beta-\mathrm{GA}$ was shown to significantly suppress the expression of surface inflammatory trigger molecules, such as CD80, CD86, and MHC class I and II. Thus, it can be established that licorice extract can be a useful adjuvant in COVID-19 infection or related complications.

- Ashwagandha: Also known as Withania somnifera, ashwagandha is a very useful herb in Indian ayurvedic system of medicine for the treatment of various diseases. Ashwagandha contains various biologically active constituents like alkaloids (isopelletierine, cuscohygrine, anaferine, anahygrine, etc.), steroidal lactones (withanolides, withaferins), and saponins. ${ }^{59}$ Ashwagandha is known to have various beneficial properties like enhancing cell-mediated immunity that helps protect 
against various infections like viral, bacterial, and fungal. The probable mode of action is increasing enzyme nitric oxide synthase in the macrophages that enhances the antimicrobial activity of the macrophages thereby enhancing the cellmediated immune (CMI) response. In a study by Purshotam et al., ${ }^{60}$ Withaferin A. extract was shown to regulate all major four pathways of innate inflammation (MAPKs and NF-KB). Although no data are available showing the benefit of ashwagandha in COVID-19 virus infections, the indirect evidence from the above studies can conclude that ashwagandha is helpful in patients having SARS-CoV-2 infection.

\section{Conclusion}

COVID-19 (SARS-CoV-2), a pandemic disease, has a high mortality rate. The COVID-19 infection generates abnormal "cytological storm" with production of multiple interleukins like IL-2, IL-6, IL-12, IL-18, etc., and inflammatory CC. All of these particles lead to microvascular thrombosis in pulmonary blood vessels and PF leading to death. So, the drugs having antiviral properties along with preventive effect on cytological storm are required. Indian herbal drugs like curcumin, amla, kalimirch, cinnamon, garlic, ginger, mulethi, etc., due to their immunomodulation and antiviral effects seem to have such potential in preventing complications.

\section{References}

1. Zhao $L$, Jha BK, Wu A, et al. Antagonism of the interferon-induced OAS-RNase $L$ pathway by murine coronavirus ns 2 protein is required for virus replication and liver pathology. Cell Host Microbe 2012;11(6):607-616. DOI: 10.1016/j.chom.2012.04.011.

2. Neuman BW, Adair BD, Yoshioka C, et al. Supramolecular architecture of severe acute respiratory syndrome coronavirus revealed by electron cryomicroscopy. JVirol 2006;80(16):7918-7928. DOI: 10.1128/ JVI.00645-06.

3. Yang H, Bartlam M, Rao Z. Drug design targeting the main protease, the Achilles' heel of coronaviruses. Curr Pharm Des 2006;12(35):45734590. DOI: $10.2174 / 138161206779010369$.

4. Coronavirus Disease 2019 (COVID-19). Updated April 19, 2020 - CDC. Source: https://www.cdc.gov/coronavirus/2019-nCoV/summary.html. Accessed 10.05.2020.

5. Yang CL, Qiu X, Zeng YK, et al. Coronavirus disease 2019: a clinical review. Eur Rev Med Pharmacol Sci 2020;24(8):4585-4596. DOI: 10.26355/eurrev_202004_21045.

6. Law HK, Cheung $\mathrm{CY}, \mathrm{Ng} \mathrm{HY}$, et al. Chemokine upregulation in SARS coronavirus infected human monocyte derived dendritic cells. Blood 2005;106(7):2366-2376. DOI: 10.1182/blood-2004-10-4166.

7. Liu J, Wu P, Gao F, et al. Novel immunodominant peptide presentation strategy: a featured HLA-A*2402-restricted cytotoxic T-lymphocyte epitope stabilized by intrachain hydrogen bonds from severe acute respiratory syndrome coronavirus nucleocapsid protein. J Virol 2010;84(22):11849-11857. DOI: 10.1128/JVI.01464-10.

8. Li G, Chen X, Xu A. Profile of specific antibodies to the SARS-associated coronavirus. N Engl J Med 2003;349(5):508-509. DOI: 10.1056/ NEJM200307313490520.

9. Fan YY, Huang ZT, Li L, et al. Characterization of SARS-CoV-specific memory $T$ cells from recovered individuals 4 years after infection. Arch Virol 2009;154(7):1093-1099. DOI: 10.1007/s00705-009-0409-6.

10. Mehta P, Mcauley D, Brown M, et al. Correspondence COVID-19: consider cytokine storm syndromes and immunosuppression. Lancet 2020;395(10229):1033-1034. DOI: 10.1016/S0140-6736(20)30628-0.

11. Li S, Jiang L, Li X, et al. Clinical and pathological investigation of patients with severe COVID-19. JCI Insight 2020;5(12):138070. DOI: 10.1172/jci.insight.138070.

12. Channappanavar R, Perlman S. Pathogenic human coronavirus infections: causes and consequences of cytokine storm and immunopathology. Semin Immunopathol 2017;39(5):529-539. DOI: 10.1007/s00281-017-0629-x.

13. Dhama K, Patel SK, Pathak M, et al. An update on SARS-CoV-2/COVID19 with particular reference to its clinical pathology, pathogenesis, immunopathology and mitigation strategies. Travel Med Infect Dis 2020;37:101755. DOI: 10.1016/j.tmaid.2020.101755.

14. Liu Q, Wang R, Qu G, et al. Gross examination report of a COVID-19 death autopsy. Fa Yi Xue Za Zhi 2020;36(1):21-23. DOI: 10.12116/j. issn.1004-5619.2020.01.005.

15. Kampf G, Todt D, Pfaender S, et al. Persistence of coronaviruses on inanimate surfaces and its inactivation with biocidal agents. J Hosp Infect 2020;104(3):246-251. DOI: 10.1016/j.jhin.2020.01.022.

16. Ho LTF, Chan KKH, Chung VCH, et al. Highlights of traditional Chinese medicine frontline expert advice in the China national guideline for COVID-19. Eur J Integr Med 2020;36:101116. DOI: 10.1016/j. eujim.2020.101116.

17. Zhang W, Du RH, Li B, et al. Molecular and serological investigation of 2019-nCoV infected patients: implication of multiple shedding routes. Emerg Microbes Infect 2020;9(1):386-389. DOI: 10.1080/22221751.2020.1729071.

18. Ruan Q, Yang K, Wang W, et al. Clinical predictors of mortality due to COVID-19 based on an analysis of data of 150 patients from Wuhan, China. Intensive Care Med 2020:1-4. DOI: 10.1007/s00134-02005991-x.

19. Taoran G, Yang H, Taisheng L, et al. Characteristics and prognostic value of peripheral blood T lymphocyte subsets in patients with severe influenza. Zhonghua Nei Ke Za Zhi 2020;59(3):200-206. DOI: 10.3760/cma.j.issn.0578-1426.2020.03.006.

20. Huang C, Wang Y, Li X, et al. Clinical features of patients infected with 2019 novel coronavirus in Wuhan, China. Lancet 2020;395(10223):497506. DOI: 10.1016/S0140-6736(20)30183-5.

21. Liu F, Li L, Xu M, et al. Prognostic value of interleukin-6, C-reactive protein, and procalcitonin in patients with COVID-19. J Clin Virol 2020;127:104370. DOI: 10.1016/j.jcv.2020.104370.

22. Wang M, Cao R, Zhang L, et al. Remdesivir and chloroquine effectively inhibit the recently emerged novel coronavirus (2019-nCoV) in vitro. Cell Res 2020;30(3):269-271. DOI: 10.1038/s41422-020-0282-0.

23. SchrezenmeierE,DörnerT.Mechanisms of action of hydroxychloroquine and chloroquine: implications for rheumatology. Nat Rev Rheumatol 2020;16(3):155-166. DOI: 10.1038/s41584-020-0372-x.

24. Jean SS, Lee PI, Hsueh PR. Treatment options for COVID-19: the reality and challenges. J Microbiol Immunol Infect 2020;53(3):436-443. DOI: 10.1016/j.jmii.2020.03.034.

25. Ford N, Vitoria M, Rangaraj A, et al. Systematic review of the efficacy and safety of antiretroviral drugs against SARS, MERS or COVID-19: initial assessment. J Int AIDS Soc 2020;23(4):e25489. DOI: 10.1002/ jia2.25489.

26. Zhu Z, Lu Z, Xu T, et al. Arbidol monotherapy is superior to lopinavir/ ritonavir in treating COVID-19. J Infect 2020;81(1):e21-e23. DOI: 10.1016/j.jinf.2020.03.060. S0163-4453(20)30188-2.

27. Furuta Y, Komeno T, Nakamura T. Favipiravir (T-705), a broad spectrum inhibitor of viral RNA polymerase. Proc Jpn Acad, Ser B, Phys Biol Sci 2017;93(7):449-463. DOI: 10.2183/pjab.93.027.

28. Gordon CJ, Tchesnokov EP, Feng JY, et al. The antiviral compound remdesivir potently inhibits RNA-dependent RNA polymerase from Middle East respiratory syndrome coronavirus. J Biol Chem 2020;295(15):4773-4779. DOI: 10.1074/jbc.AC120.013056.

29. Tocilizumab (Rx). Source: https://reference.medscape.com/drug/ actemra-tocilizumab-999419. Accessed on 10.05.2020.

30. Wang H, Liu B, Tang Y, et al. Improvement of sepsis prognosis by ulinastatin: a systematic review and meta-analysis of randomized controlled trials. Front Pharmacol 2019;10:1370. DOI: 10.3389/ fphar.2019.01370.

31. Zhang X, Zhu Z, Jiao W, et al. Ulinastatin treatment for acute respiratory distress syndrome in China: a meta-analysis of randomized controlled trials. BMC Pulm Med 2019;19(1):196. DOI: 10.1186/s12890-019-0968-6.

32. Karnad DR, Bhadade R, Verma PK, et al. Intravenous administration of ulinastatin (human urinary trypsin inhibitor) in severe sepsis: 
a multicenter randomized controlled study. Intensive Care Med 2014;40(6):830-838. DOI: 10.1007/s00134-014-3278-8.

33. Stockman LJ, Bellamy R, Garner P. SARS: Systematic review of treatment effects. PLoS Med 2006;3(9):e343. DOI: 10.1371/journal. pmed.0030343.

34. Hemming VG. Use of intravenous immunoglobulins for prophylaxis or treatment of infectious diseases. Clin Diagn Lab Immunol 2001;8(5):859-863. DOI: 10.1128/CDLI.8.5.859-863.2001.

35. Duan K, Liu B, Li C, et al. Effectiveness of convalescent plasma therapy in severe COVID-19 patients. PNAS 2020;117(17):9490-9496. DOI: 10.1073/pnas.2004168117.

36. Heidary F, Gharebaghi R. Ivermectin: a systematic review from antiviral effects to COVID-19 complementary regimen. J Antibiot (Tokyo) 2020;73(9):593-602. DOI: 10.1038/s41429-020-0336-z.

37. Caly L, Druce JD, Catton MG, et al. The FDA-approved drug ivermectin inhibits the replication of SARS-CoV-2 in vitro. Antivir Res 2020;178:104787. DOI: 10.1016/j.antiviral.2020.104787.

38. Wagstaff KM, Sivakumaran $\mathrm{H}$, Heaton $\mathrm{SM}$, et al. Ivermectin is a specific inhibitor of importin $\alpha / \beta$-mediated nuclear import able to inhibit replication of HIV-1 and dengue virus. Biochem J 2012;443:851-856. DOI: 10.1042/BJ20120150.

39. Li T. Diagnosis and clinical management of severe acute respiratory syndrome Coronavirus 2 (SARS-CoV-2) infection: an operational recommendation of Peking Union Medical College Hospital (V2.0). Working Group of 2019 Novel Coronavirus, Peking Union Medical College Hospital. Emerg Microbes Infect 2020;9(1):582-585. DOI: 10.1080/22221751.2020.1735265.

40. Patra JK, Das G, Bose S, et al. Star anise (Illicium verum): chemical compounds, antiviral properties, and clinical relevance. Phytother Res 2020;34(6):1-20. DOI: 10.1002/ptr.6614.

41. Li T, Peng T. Traditional Chinese herbal medicine as a source of molecules with antiviral activity. Antivir Res 2013;97(1):1-9. DOI: 10.1016/j.antiviral.2012.10.006.

42. Ren Y, Yao MC, Huo XQ, et al. Study on treatment of "cytokine storm" by anti-2019-nCoV prescriptions based on arachidonic acid metabolic pathway [in Chinese]. Zhongguo Zhong Yao Za Zhi 2020;45(6):12251231. DOI: 10.19540/j.cnki.cjcmm.20200224.405.

43. Sornpet B, Potha T, Tragoolpua Y, et al. Antiviral activity of five Asian medicinal pant crude extracts against highly pathogenic H5N1 avian influenza virus. Asian Pac J Trop Med 2017;10(9):871-876. DOI: 10.1016/j.apjtm.2017.08.010.

44. Jobin C, Bradham CA, Russo MP, et al. Curcumin blocks cytokinemediated NF-kappa B activation and proinflammatory gene expression by inhibiting inhibitory factor I-kappa B kinase activity. J Immunol 1999;163:3474-3483.

45. Liu G, Xiong $S$, Xiang YF, et al. Antiviral activity and possible mechanisms of action of pentagalloylglucose (PGG) against influenza A virus. Arch Virol 2011;156(8):1359-1369. DOI: 10.1007/s00705-011-0989-9.

46. Alhazmi MI. Molecular docking of selected phytocompounds with H1N1 Proteins. Bioinformation 2015;11(4):196-202. DOI: 10.6026/97320630011196.
47. Lane T, Anantpadma M, Freundlich JS, et al. The natural product eugenol is an inhibitor of the ebola virus in vitro. Pharm Res 2019;36(7):104. DOI: 10.1007/s11095-019-2629-0.

48. Yeh CF, Chang JS, Wang KC, et al. Water extract of Cinnamomum cassia Blume inhibited human respiratory syncytial virus by preventing viral attachment, internalization, and syncytium formation. J Ethnopharmacol 2013;147(2):321-326. DOI: 10.1016/ j.jep.2013.03.010.

49. $\operatorname{Kim~N}$, Do J, Bae JS, et al. Piperlongumine inhibits neuroinflammation via regulating NF-KB signaling pathways in lipopolysaccharidestimulated BV2 microglia cells. J Pharmacol Sci 2018;137(2):195-201. DOI: 10.1016/j.jphs.2018.06.004.

50. El-Saber Batiha G, Magdy Beshbishy A, G Wasef L, et al. Chemical constituents and pharmacological activities of garlic (Allium sativum L.): a review. Nutrients 2020;12(3):872. DOI: 10.3390/nu12030872.

51. Burian JP, Sacramento LVS, Carlos IZ. Fungal infection control by garlic extracts (Allium sativum L.) and modulation of peritoneal macrophages activity in murine model of sporotrichosis. Braz J Biol 2017;77(4):848-855. DOI: 10.1590/1519-6984.03716.

52. Mohajer Shojai T, Ghalyanchi Langeroudi A, Karimi V, et al. The effect of Allium sativum (garlic) extract on infectious bronchitis virus in specific pathogen free embryonic egg. Avicenna J Phytomed 2016;6(4):458-267.

53. Fukao $\mathrm{H}$, Yoshida $\mathrm{H}$, Tazawa $\mathrm{Y}$, et al. Antithrombotic effects of odorless garlic powder both in vitro and in vivo. Biosci Biotechnol Biochem 2007;71(1):84-90. DOI: 10.1271/bbb.60380.

54. Podlogar JA, Verspohl EJ. Antiinflammatory effects of ginger and some of its components in human bronchial epithelial (BEAS-2B) cells. Phytother Res 2012;26(3):333-336. DOI: 10.1002/ptr.3558.

55. Çifci A, Tayman C, Yakut Hi, et al. Ginger (Zingiber officinale) prevents severe damage to the lungs due to hyperoxia and inflammation. Turk J Med Sci 2018;48(4):892-900. DOI: 10.3906/sag-1803-223.

56. Song J, Fan $\mathrm{HJ}, \mathrm{Li} \mathrm{H}$, et al. Zingerone ameliorates lipopolysaccharideinduced acute kidney injury by inhibiting Toll-like receptor 4 signaling pathway. Eur J Pharmacol 2016;772:108-114. DOI: 10.1016/j. ejphar.2015.12.027.

57. Cinatl J, Morgenstern B, Bauer G, et al. Glycyrrhizin, an active component of liquorice roots, and replication of SARS-associated coronavirus. Lancet 2003;361(9374):2045-2046. DOI: 10.1016/S01406736(03)13615-X.

58. Kim ME, Kim HK, Kim DH, et al. 18ß-Glycyrrhetinic acid from licorice root impairs dendritic cells maturation and Th1 immune responses. Immunopharmacol Immunotoxicol 2013;35(3):329-335. DOI: 10.3109/08923973.2013.768636.

59. Mishra LC, Singh BB, Dagenais S. Scientific basis for the therapeutic use of Withania somnifera (ashwagandha): a review. Altern Med Rev 2000;5(4):334-346.

60. Purushotham PM, Kim JM, Jo EK, et al. Withanolides against TLR4activated innate inflammatory signalling pathways: a comparative computational and experimental study. Phytother Res 2017;31(1):152163. DOI: 10.1002/ptr.5746. 